\title{
A Property of the Structure Constants of Finite Dimensional Compact Simple Lie Algebras
}

\author{
M. L. Metha ${ }^{1}$, J. M. Normand ${ }^{1}$, and V. Gupta ${ }^{2}$ \\ 1 SPh-T, C.E.N. Saclay, F-91191 Gif-sur-Yvette Cedex, France \\ 2 Tata Institute of Fundamental Research, Bombay 400005, India
}

\begin{abstract}
We consider products of structure constants of a finite-dimensional compact simple Lie algebra, in which all indices except a few are contracted in pairs. We prove that such a product is zero if only one index is free, is proportional to the Cartan-Killing tensor if two indices are free and is proportional to a structure constant itself if three indices are free. For $\mathrm{SU}(n)$, $n \geqq 3$ we also consider products of usual $d$ (related to the anti-commutator) and structure constants $f$. The results for one and two free indices are still valid. For three free indices the product is proportional to either an $f$ or a $d$ according to whether the number of $f$ 's in the product is odd or even.
\end{abstract}

\section{Introduction}

In quantum chromodynamics (QCD), the various fields carry colour and the interaction vertices depend explicitly on quantities connected with the colour group $\mathrm{SU}(3)_{c}$, which is gauged to produce the interactions. In particular, the colour dependence of the bare triple gluon and ghost-gluon vertices is given by the structure constants $c_{i j k}$ of the colour group (forgetting for the moment about the contravariant or covariant character of the indices). The question then arises about the colour dependence of the vertices in higher loop orders. Is it still given by $c_{i j k}$ as above, or are there additional terms with a different dependence on the colour indices? This question is important from the viewpoint of having a renormalizable theory. The point is that unless the counterterms required for these vertices in higher orders depend only on $c_{i j k}$, such theories will not be renormalizable in the usual way. Fortunately, using charge conjugation invariance, one can show [1] that the proper triple gluon vertex depends solely on $c_{i j k}$. However, this proof does not work for the ghost-gluon vertex. Instead one can use the Slavnov-Taylor identity [2] connecting the two vertices to show [1] that in two momentum subtraction schemes [3] the divergent part of the ghost-gluon vertex depends only on $c_{i j k}$, though finite parts with a different colour dependence are not ruled out. Much before this proof was found, one was led to speculate that there may be a 
mathematical property of the structure constants themselves which would ensure that the proper ghost-gluon vertex is always proportional to $c_{i j k}$.

In any higher order contribution to the ghost-gluon vertex, the colour dependent part will factor out and will be a combination of the structure constants with three free colour indices. Moreover, since any higher order diagram is constructed from the elementary vertices connected by propagators, and since the ghost fields do not couple directly to quarks, the dummy indices will be contracted pairwise. Thus, the general form of the colour dependence expected is

$$
T_{i j k}=c_{i \ldots} c_{j \ldots} c_{k \ldots} \ldots c_{\ldots} c_{\ldots} .
$$

The dots represent dummy indices which are contracted pairwise only. Our considerations include the $T_{i j k}$ in which $i j$, $i k$ or $j k$ may belong to the same $c$. Elementary counting tells us that the number of $c$ 's in Eq. (1.1) must be odd. In words: a combination of an odd number of $c$ 's with three free indices is a number times a single $c$, provided the dummy indices in the combination are contracted pairwise.

Apart from the original motivation from QCD, it is interesting in its own right to prove that all such $T_{i j k}$ are proportional to $c_{i j k}$. We prove this not only for $\mathrm{SU}(3)$ but for all compact simple Lie algebras. We also prove that contracted tensor products of $c$ 's with one and two free indices are, respectively, zero and proportional to the Cartan-Killing form. Two proofs are given; one pleasing to the mathematically minded (Sects. 2-4) and the other which might appeal to the physicists (Sect. 5). In the special case of $\mathrm{SU}(n), n \geqq 3$ these results are extended using the first proof to the contracted tensor products containing $f_{i j k}$ (proportional to $c_{i j k}$ ) and $d_{i j k}$. Some numerical results for $\mathrm{SU}(3)$ are given in Sect. 6.

\section{Invariance of the Structure Constants under the Adjoint Representation}

In this section we recall some well known results which will be relevant for us.

Let $u, v, \ldots$ be the elements of a finite-dimensional Lie Algebra $\mathscr{A}$ and $[u, v]$ their anti-symmetric Lie product. The Jacobi identity

$$
[u,[v, w]]+[v,[w, u]]+[w,[u, v]]=0
$$

tells us that the adjoint operator $\Delta_{u}$ defined for every $v$ in $\mathscr{A}$ by

$$
\Delta_{u} v=[u, v],
$$

gives a representation of the algebra $\mathscr{A}$ into itself: the so-called adjoint representation. The Cartan-Killing form is defined by

$$
g(u, v)=\operatorname{Tr}\left(\Delta_{u} \Delta_{v}\right) .
$$

Let $\mathscr{G}$ be the simply connected Lie group associated with $\mathscr{A} ; u$ in $\mathscr{A}$ corresponds to $\exp u$ in $\mathscr{G}$. The adjoint representation $\Delta \equiv \Delta(\mathscr{G})$ of $\mathscr{G}$ is defined by the formal power series

$$
\exp \Delta_{u}=\sum_{n=0}^{\infty} \frac{1}{n !}\left(\Delta_{u}\right)^{n}
$$


It is known ${ }^{1}$ that the Lie product is invariant under the adjoint representation $\Delta$, i.e. for every $u, v$ and $w$ in $\mathscr{A}$

$$
\left[\exp \left(\Delta_{u}\right) v, \exp \left(\Delta_{u}\right) w\right]=\exp \left(\Delta_{u}\right)[v, w] .
$$

With respect to any basis $\left(e_{i}\right)$ of $\mathscr{A}$, the structure constants $c_{i j}{ }^{k}$ are defined by

$$
\left[e_{i}, e_{j}\right]=c_{i j}{ }^{k} e_{k} .
$$

Here and in what follows, summation over repeated indices will be understood. The matrix elements of $\Delta_{e_{i}}$ are thus equal to the structure constants and the Cartan-Killing form reads

$$
g_{i j} \equiv g\left(e_{i}, e_{j}\right)=c_{i k}{ }^{l} c_{j l}{ }^{k} .
$$

From now on we will be concerned only with the semi-simple Lie algebras over the complex field. They are characterized by a non-degenerate Cartan-Killing form. In these algebras one can lower or raise the indices at will using the nonsingular metric tensor $\left(g_{i j}\right)$ and its inverse $\left(g^{i j}\right)$. Tensorial sequences which are related by the above procedure are said to be equivalent and an equivalent class is called a tensor, the components of which are the tensorial sequences with a definite tensorial (contravariant or covariant) character. For instance, $\left(c_{i j}{ }^{k}\right)$ are particular components of a tensor $c^{(3)}$ of order three. Its completely covariant components $\left(c_{i j k}\right)$ are known to be totally anti-symmetric in the three indices. This is an intrinsic property of the structure constant tensor $c^{(3)}$. Similarly, $\left(g_{i j}\right)$ are the covariant components of a tensor $g^{(2)}$ of order two which is symmetric.

Under a change of basis

$$
e_{i}^{\prime}=e_{j} M_{i}^{j},
$$

where $\left(M_{i}^{j}\right)$ is any non-singular matrix, the structure constants and the metric tensor transform as tensorial sequences of order three and two respectively. For example,

$$
\begin{gathered}
c_{i j k}^{\prime}=M_{i}^{l} M_{j}^{m} M_{k}^{n} c_{l m n}, \quad c_{i j}^{\prime k}=M_{i}^{l} M_{j}^{m}\left(M^{-1}\right)_{n}^{k} c_{l m}{ }^{n}, \\
g_{i j}^{\prime}=M_{i}^{l} M_{j}^{m} g_{l m} .
\end{gathered}
$$

We now restrict ourselves to changes of basis in $\mathscr{A}$ generated by the adjoint representation $\Delta$,

$$
e_{i}^{\prime}=e_{j}\left(\exp \Delta_{u}\right)_{i}^{j}
$$

for any $u$ in $\mathscr{A}$. It follows from Eq. (2.5) that the structure constants form an invariant tensorial sequence, i.e.

$$
c_{i j}^{\prime}{ }^{k}=\left(\exp \Delta_{u}\right)_{i}^{l}\left(\exp \Delta_{u}\right)_{j}^{m}\left(\exp -\Delta_{u}\right)_{n}^{k} c_{l m}{ }^{n}=c_{i j}{ }^{k} .
$$

1 Expanding the exponential series in the left hand side of Eq. (2.5), and using induction on $n$ and the Jacobi identity (2.1) to prove $\sum_{\substack{p, q=0 \\ p+q=n}}^{\infty} \frac{n !}{p ! q !}\left[\left(\Delta_{u}\right)^{p} v,\left(\Delta_{u}\right)^{q} w\right]=\left(\Delta_{u}\right)^{n}[v, w]$, one gets the result 
Since the metric tensor $\left(g_{i j}\right)$ is given in terms of the structure constants [Eq. (2.7)], it also forms an invariant tensorial sequence, i.e.

$$
g_{i j}^{\prime}=\left(\exp \Delta_{u}\right)_{i}^{l}\left(\exp \Delta_{u}\right)_{j}^{m} g_{l m}=g_{i j} .
$$

Hence $c^{(3)}$ and $g^{(2)}$ are two invariant tensors under the adjoint representation $\Delta$. This property plays a central role in proving our main results.

\section{Contracted Tensor Products of Structure Constant Tensors}

Let

$$
T^{(n)}=c^{(3)} \otimes c^{(3)} \otimes \ldots \otimes c^{(3)}
$$

be a contracted tensor product of $p$ tensors $c^{(3)}$ in which $n$ indices are free. Elementary counting implies that $n+p$ is even. For example, $g^{(2)}$ is itself a $T^{(2)}$ tensor, and for $p=5$ :

$$
T_{i}=c_{i j k} c_{m}^{j l} c_{n q}^{r} c_{r}^{k} c_{l}^{q n}
$$

will represent the components of a $T^{(1)}$.

We now prove that for a finite-dimensional compact simple Lie algebra $\mathscr{A}$ one has:

$$
T^{(1)}=0, \quad T^{(2)}=\lambda g^{(2)}, \quad T^{(3)}=\mu c^{(3)},
$$

where $\lambda$ and $\mu$ are constants (possibly zero) which depend upon the tensor considered.

The components of $T^{(n)}$ are a tensorial sequence of order $n$ transforming according to the $n^{\text {th }}$ power of the adjoint representation $\Delta$. Since from Sect. 2 the tensor $c^{(3)}$ is invariant under $\Delta$, the same holds true for any $T^{(n)}$.

For compact Lie groups any finite-dimensional representation is either irreducible or completely reducible. Therefore, the invariant tensor $T^{(n)}$, as any other invariant, transforms according to the unit representation of $\mathscr{G}$ (WignerEckart theorem). Hence, to find what $T^{(n)}$ can be, we have to answer the question: how many times does the unit representation occur in the $n^{\text {th }}$ power of $\Delta$ ?

From now on we consider only compact simple Lie groups. For these groups it is known that $\Delta$ is irreducible and different from the unit representation. Also that the unit representation occurs once in $D_{1} \otimes D_{2}^{*}$ if and only if the two irreducible representations $D_{1}$ and $D_{2}$ are equivalent [4]. Moreover, $\Delta$ is real $[5,6], \Delta \approx \Delta^{*}$. Therefore, the unit representation occurs:

(i) zero times in $\Delta$,

(ii) once in $\Delta \otimes \Delta$, and

(iii) as many times in $\Delta \otimes \Delta \otimes \Delta$ as $\Delta$ occurs in $\Delta \otimes \Delta$.

It is verified in the appendix that $\Delta$ occurs twice in $\Delta \otimes \Delta$ for the algebra $A_{n}$ (isomorphic to the algebra of $\mathrm{SU}(n+1)), n \geqq 2$, and once for every other compact simple Lie algebra including the five exceptional ones.

Thus:

(i) $T^{(1)}=0$.

(ii) All $T^{(2)}$ are proportional to one of them, say the Cartan-Killing tensor $g^{(2)}$. 
(iii) For all compact simple Lie algebras, except $A_{n}, n \geqq 2$, all $T^{(3)}$ are proportional to one of them, say the structure constant tensor $c^{(3)}$ itself. This ends the proof of Eqs. (3.3) for these algebras.

(iv) For $A_{n}, n \geqq 2$, one needs further arguments to show that $T^{(3)}$ is proportional to $c^{(3)}$, since there are two linearly independent third order invariant tensors under $\Delta$. This is done in the next section.

\section{The Special Case of $S U(n), n \geqq 3$}

The Lie algebra of $\mathrm{SU}(n)$ is isomorphic to the $\left(n^{2}-1\right)$-dimensional Lie algebra $\mathscr{A}$ over the real field of $n \times n$ traceless hermitian matrices, the Lie product being defined by $-i$ times the commutator. Without loss of generality, we choose the commonly used [7] basis $\left(V_{i}\right)$ of $\mathscr{A}$ normalized such that:

$$
\operatorname{Tr}\left(V_{i} V_{j}\right)=2 \delta_{i j}
$$

The commutator and the anti-commutator of two $V$ 's are then given by:

$$
\begin{gathered}
{\left[V_{i}, V_{j}\right]=2 i f_{i j k} V_{k},} \\
\left\{V_{i}, V_{j}\right\}=\frac{4}{n} \delta_{i j} \mathbf{1}+2 d_{i j k} V_{k} .
\end{gathered}
$$

The structure constants $f_{i j k}$ are related to the c's considered previously by

$$
f_{i j k}=\frac{1}{2} c_{i j}{ }^{k}=-\frac{1}{8 n} c_{i j k} \text {. }
$$

The $f_{i j k}$ and the $d_{i j k}$ are, respectively, completely anti-symmetric and symmetric in $i, j$, and $k$. Both of them are real.

The adjoint representation of $\mathrm{SU}(n)$ corresponds to changes of basis in $\mathscr{A}$ defined for all $U$ in $\mathrm{SU}(n)$ by:

$$
V_{i}^{\prime}=U V_{i} U^{-1}
$$

Thus, the tensorial sequences $\left(f_{i j k}\right)$ and $\left(d_{i j k}\right)$ are seen to be invariant under the adjoint representation. They are the components of the two linearly independent third order tensors $f^{(3)}$ and $d^{(3)}$. Consequently, any invariant tensor $T^{(3)}$ is a linear combination of $f^{(3)}$ and $d^{(3)}$.

To distinguish between $f^{(3)}$ and $d^{(3)}$ we examine the law of transformation of these tensors under the change of basis in $\mathscr{A}$ defined by the complex conjugation of the basis matrices $V_{i}$. Since $f_{i j k}$ and $d_{i j k}$ are real, taking the complex conjugate of Eqs. (4.2) and (4.3) one finds that $f^{(3)}$ changes sign while $d^{(3)}$ remains invariant:

$$
V_{i} \rightarrow V_{i}^{*} \Rightarrow f^{(3)} \rightarrow-f^{(3)}, \quad d^{(3)} \rightarrow d^{(3)} .
$$

It follows that $T^{(3)}$ defined as the contracted tensor product of an odd number of tensors $f^{(3)}$ changes sign under complex conjugation of the $V_{i}$, and therefore is a multiple of $f^{(3)}$. This completes the proof of Eq. (3.3).

More generally, let $T_{a p}^{(n)}$ be a tensor of order $n$ defined as a contracted tensor product of $q$ tensors $d^{\left(3^{3}\right)}$ and $p$ tensors $f^{(3)}$. Elementary counting implies that 


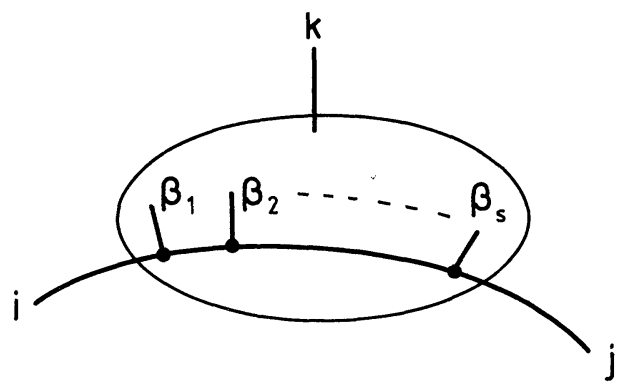

Fig. 1. A path starting at line $i$ and ending in line $j$ associated with the expression for $T_{i j k}$ given in Eq. (5.1)

$n+p+q$ is even. This tensor $T_{q p}^{(n)}$ is invariant under the adjoint representation of $\mathrm{SU}(n)$, and following the same arguments as above yields for all $q$ and $p$ :

$$
\begin{gathered}
T_{q p}^{(1)}=0, \quad T_{q p}^{(2)}=\lambda g^{(2)}, \\
T_{q p}^{(3)}= \begin{cases}\mu d^{(3)} & \text { if } q \text { is odd and } p \text { even } \\
v f^{(3)} & \text { if } q \text { is even and } p \text { odd },\end{cases}
\end{gathered}
$$

where $\lambda, \mu$, and $v$ are constants (possibly zero) depending upon the tensor considered.

\section{Second Proof of the Nature of $T^{(n)}$ for $n \leqq 3$}

This proof is based on the matrix representation of the adjoint operator. Let us denote by $\Delta_{i}$ the matrix of $\Delta_{e_{i}}$ with respect to a basis $\left(e_{i}\right)$ of $\mathscr{A}$. Without loss of generality, for compact simple Lie algebras we choose $\left(g_{i j}\right)$ to be proportional to the unit matrix. Then $c_{i j k}=\left(\Delta_{i}\right)_{k j}=\left(\Delta_{j}\right)_{i k}=\left(\Delta_{k}\right)_{j i}$ follows from the complete antisymmetry of the $c_{i j k}$. This is used below to express products of $c$ 's in terms of matrix products.

A product of any number of $c$ 's with certain contracted indices can be represented by a trivalent graph. Apart from a sign, a $c$ is denoted by a point and its indices by lines radiating from it, a line joining two points corresponds to a contracted index. Thus $T_{i j k}$ is represented by a trivalent graph with three outgoing lines marked $i, j$, and $k$. It is sufficient to consider connected graphs. One can then always trace a path starting at line $i$ and ending in line $j$, see Fig. 1. Therefore, $T_{i j k}$ can always be written as:

$$
T_{i j k}=M_{i j} c_{\alpha_{1} \alpha_{2} \alpha_{3}} \ldots c_{\alpha_{r-2} \alpha_{r-1} \alpha_{r}}
$$

where $M_{i j}$ is the $(i, j)$ matrix element of the matrix

$$
M=\Delta_{\beta_{1}} \Delta_{\beta_{2}} \ldots \Delta_{\beta_{s}} .
$$

By definition all the indices $\alpha$ and $\beta$, except $k$ which is one of them, are pairwise contracted. Since the graph is connected, there is at least one index in the set $\left\{\beta_{1}, \ldots, \beta_{s}\right\}$, call it $\beta$, which occurs in the set $\left\{\alpha_{1}, \ldots, \alpha_{r}\right\}$. Denote the unique structure constant which carries the index $\beta$ in Eq. (5.1) by $c_{\alpha \alpha^{\prime} \beta}$. Now using the 
obvious matrix relation

$$
c_{\alpha \alpha^{\prime} \beta} \Delta_{\beta}=\Delta_{\alpha} \Delta_{\alpha^{\prime}}-\Delta_{\alpha^{\prime}} \Delta_{\alpha}
$$

again and again, we can absorb all the $c$ 's in Eq. (5.1). Then $T_{i j k}$ can be written as the $(i, j)$ matrix element of a linear combination of matrices

$$
T_{i j k}=\left(M^{\prime}+M^{\prime \prime}+\ldots\right)_{i j},
$$

where $M^{\prime}, M^{\prime \prime}, \ldots$ are, up to a sign, matrices of the type defined by Eq. (5.2). This time $\Delta_{k}$ occurs in each of them.

Given Eq. (5.4), it is sufficient to show that any such matrix

$$
M_{k}=\Delta_{\beta_{1}} \ldots \Delta_{\beta_{m}} \Delta_{k} \Delta_{\beta_{m+2}} \ldots \Delta_{\beta_{p}},
$$

$p$ odd, all indices $\beta$ except $k$ being contracted in pairs, is proportional to $\Delta_{k}$. Using

$$
\left[X, Y_{1} Y_{2} \ldots Y_{p}\right]=\left[X, Y_{1}\right] Y_{2} \ldots Y_{p}+Y_{1}\left[X, Y_{2}\right] \ldots Y_{p}+\ldots+Y_{1} Y_{2} \ldots\left[X, Y_{p}\right],
$$

the commutator $\left[\Delta_{i}, M_{j}\right]$ can be written as a sum of terms. One of them is:

$$
\Delta_{\beta_{1}} \ldots\left[\Delta_{i}, \Delta_{j}\right] \ldots \Delta_{\beta_{p}}=c_{i j k} \Delta_{\beta_{1}} \ldots \Delta_{k} \ldots \Delta_{\beta_{p}}=c_{i j k} M_{k},
$$

while the other terms are paired to have a zero sum. Indeed, for any contracted index $\beta$ which occurs twice in the right hand side of Eq. (5.5), there are two terms

$$
\ldots\left[\Delta_{i}, \Delta_{\beta}\right] \ldots \Delta_{\beta} \ldots+\ldots \Delta_{\beta} \ldots\left[\Delta_{i}, \Delta_{\beta}\right] \ldots=c_{i \beta \gamma}\left(\ldots \Delta_{\gamma} \ldots \Delta_{\beta} \ldots+\ldots \Delta_{\beta} \ldots \Delta_{\gamma} \ldots\right),
$$

which sum to zero owing to the anti-symmetry of $c_{i \beta \gamma}$. Thus one finds:

$$
\left[\Delta_{i}, M_{j}\right]=c_{i j k} M_{k} .
$$

The number of linearly independent solutions $M_{i}$ of this equation is the number of times the adjoint representation $\Delta$ occurs in the reduction of $\Delta \otimes \Delta$. The arguments of Sects. 3 and 4 above then complete the proof that $M_{k}$ is proportional to $\Delta_{k}$.

It then follows that

$$
T_{i j k}=\mu c_{i j k},
$$

from which one can easily deduce the results for one and two free indices, i.e.

$$
\begin{gathered}
T_{i}=T_{i j k} \delta_{j k}=\mu c_{i j j}=0, \\
T_{i j}=T_{i k l} c_{j l k}=\lambda \delta_{i j} .
\end{gathered}
$$

\section{Some Numerical Results for $\boldsymbol{T}_{\boldsymbol{i} j \mathrm{k}}$ in the Case of SU(3)}

Instead of $\Delta_{i}$, we use the proportional matrix $F_{i}$, the $(k, j)$ matrix element of which is $f_{i j k}$. In the graphical representation of $T_{i j k}$ with at least three $f$ 's there always exists a closed path, see Fig. 2. Then, the same arguments as in Sect. 5 allow us to write any $T_{i j k}$ as a linear combination of traces of products of $F$ 's. In the compact notation of indicating $F_{i}$ by the index $i$ it carries, we write:

$$
\operatorname{Tr}\left(F_{i} \ldots F_{j} \ldots F_{k} \ldots\right) \equiv \operatorname{Tr}(i \ldots j \ldots k \ldots) .
$$




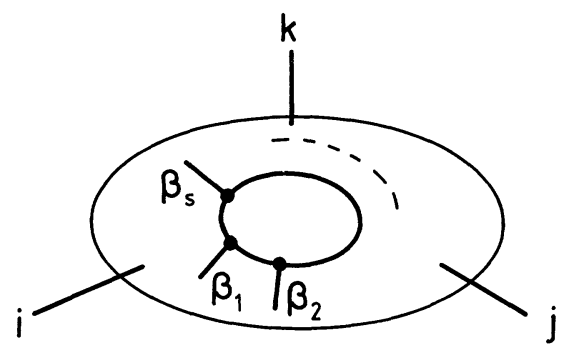

Fig. 2. A closed path which allows us to write any $T_{i j k}$ as $\operatorname{Tr}\left(\Delta_{\beta_{1}} \Delta_{\beta_{2}} \ldots \Delta_{\beta_{s}}\right)$ times a product of $c$ 's

Table 1. Traces containing products of nine $F$ s for $\mathrm{SU}(3)$. Fach is proportional to $f_{i j k}$. The constant of proportionality is given in the second column

\begin{tabular}{|c|c|}
\hline $\operatorname{Tr}(i j k l \quad m n l m n)$ & 0 \\
\hline $\operatorname{Tr}(i j l \quad k \quad m n l \quad m n)$ & 0 \\
\hline 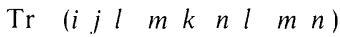 & $27 / 4$ \\
\hline 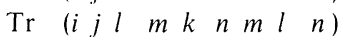 & $189 / 16$ \\
\hline $\operatorname{Tr}(i l j \quad m k n l m n)$ & $9 / 4$ \\
\hline $\operatorname{Tr}\left(\begin{array}{llllll}i l j & m k & n & m l & n\end{array}\right)$ & $225 / 16$ \\
\hline $\operatorname{Tr}\left(\begin{array}{lllllllllll}i & m & n & k & m & n\end{array}\right)$ & $27 / 4$ \\
\hline $\operatorname{Tr} \quad\left(\begin{array}{lllllllll}i & j & l & m & n & k & l & n & m\end{array}\right)$ & $27 / 4$ \\
\hline 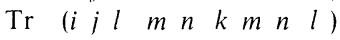 & 0 \\
\hline 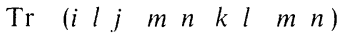 & $9 / 2$ \\
\hline 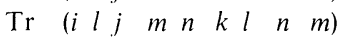 & $-\quad 9 / 4$ \\
\hline 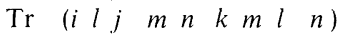 & $9 / 4$ \\
\hline 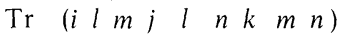 & $-9 / 2$ \\
\hline $\operatorname{Tr}\left(\begin{array}{llllllll}i & l & m & j & n & l & k & m\end{array}\right)$ & 0 \\
\hline
\end{tabular}

For products of two and three $f$ 's the results are well-known

$$
\begin{gathered}
f_{i k l} f_{j l k}=-3 \delta_{i j}=\operatorname{Tr}(i j), \\
f_{i l m} f_{j m n} f_{k n l}=\frac{3}{2} f_{i j k}=-\operatorname{Tr}(i j k) .
\end{gathered}
$$

All the products of five $f$ 's can be reduced to a product of three or less $f$ 's by using Eqs. (6.2) and (6.3).

All the products of seven f's can be expressed in terms of products of a fewer number of $f$ 's and

$$
\begin{gathered}
\operatorname{Tr}(i j l m k l m)=0, \\
\operatorname{Tr}(i l j m k l m)=36 f_{i j k} .
\end{gathered}
$$

On a systematic study of the traces of nine $f$ 's we find that one can express them all in terms of a set of fourteen traces and products of a fewer number of $f$ 's. The values of these fourteen traces are given in Table 1. Traces containing seven and nine $f$ 's were calculated on a computor using the explicit numerical values of the $f_{i j k}$. 


\section{Appendix - Number of Times the Adjoint Representation $\Delta$ Occurs in $\Delta \otimes \Delta$}

As we could not find a convenient reference, we worked out the reduction of $\Delta \otimes \Delta$ into its irreducible components. We list the result for all compact simple Lie algebras. An irreducible representation is characterised by its highest weight $\mathbf{M}=\sum_{i} \lambda_{i} \pi_{i}$ expressed in terms of the fundamental dominant weights $\pi_{i}$. In labelling these $\pi_{i}$ we have adopted the notation of Cartan (see for example, Refs. [5] and [6])

$$
\begin{aligned}
& A_{1}: \quad 2 \pi_{1} \otimes 2 \pi_{1}=4 \pi_{1} \oplus 2 \pi_{1} \oplus 0, \\
& \text { dimensions } 3 \times 3=5+3+1 \text {. } \\
& A_{2}: \quad\left(\pi_{1}+\pi_{2}\right) \otimes\left(\pi_{1}+\pi_{2}\right) \\
& =\left(2 \pi_{1}+2 \pi_{2}\right) \oplus 3 \pi_{1} \oplus 3 \pi_{2} \oplus\left(\pi_{1}+\pi_{2}\right) \oplus\left(\pi_{1}+\pi_{2}\right) \oplus 0, \\
& \text { dimensions } 8 \times 8=27+10+10+8+8+1 \text {. } \\
& A_{n}, n \geqq 3,\left(D_{3}=A_{3}\right):\left(\pi_{1}+\pi_{n}\right) \otimes\left(\pi_{1}+\pi_{n}\right) \\
& =\left(2 \pi_{1}+2 \pi_{n}\right) \oplus\left(2 \pi_{1}+\pi_{n-1}\right) \oplus\left(\pi_{2}+2 \pi_{n}\right) \oplus\left(\pi_{2}+\pi_{n-1}\right) \\
& \oplus\left(\pi_{1}+\pi_{n}\right) \oplus\left(\pi_{1}+\pi_{n}\right) \oplus 0 \text {, } \\
& \text { dimensions } n(n+2) \times n(n+2) \\
& =\frac{1}{4} n(n+1)^{2}(n+4)+\frac{1}{4} n(n-1)(n+2)(n+3) \\
& +\frac{1}{4} n(n-1)(n+2)(n+3)+\frac{1}{4}(n+1)^{2}\left(n^{2}-4\right) \\
& +n(n+2)+n(n+2)+1 \text {. } \\
& \left(A_{n}=\mathrm{SU}(n+1), n \geqq 1\right) \text {. } \\
& =\frac{1}{3}\left(n^{2}-1\right)(2 n+1)(2 n+3)+\frac{1}{2} n(n-1)(2 n+1)(2 n+3) \\
& +n(2 n+3)+\frac{1}{6} n(n-1)(2 n-1)(2 n+1)+n(2 n+1)+1 \text {. }
\end{aligned}
$$

$C_{n}, n \geqq 2,\left(B_{2}=C_{2}\right): 2 \pi_{1} \otimes 2 \pi_{1}=4 \pi_{1} \oplus\left(2 \pi_{1}+\pi_{2}\right) \oplus 2 \pi_{2} \oplus 2 \pi_{1} \oplus \pi_{2} \oplus 0$,

dimensions $n(2 n+1) \times n(2 n+1)$

$$
\begin{aligned}
= & \frac{1}{6} n(n+1)(2 n+1)(2 n+3)+\frac{1}{2} n(n-1)(2 n+1)(2 n+3) \\
& +\frac{1}{3} n(n-1)(2 n-1)(2 n+3)+n(2 n+1)+(n-1)(2 n+1)+1 .
\end{aligned}
$$

$D_{4}: \quad \pi_{4} \otimes \pi_{4}=2 \pi_{4} \oplus\left(\pi_{1}+\pi_{2}+\pi_{3}\right) \oplus 2 \pi_{3} \oplus 2 \pi_{1} \oplus \pi_{4} \oplus 0$,

dimensions $28 \times 28=300+350+35+70+28+1$.

$D_{5}: \quad \pi_{4} \otimes \pi_{4}=2 \pi_{4} \oplus\left(\pi_{3}+\pi_{5}\right) \oplus 2 \pi_{3} \oplus\left(\pi_{1}+\pi_{2}\right) \oplus \pi_{4} \oplus 0$,

dimensions $45 \times 45=770+945+54+210+45+1$. 


$$
\begin{array}{ll}
D_{n}, n \geqq 6: & \pi_{4} \otimes \pi_{4}=2 \pi_{4} \oplus\left(\pi_{3}+\pi_{5}\right) \oplus 2 \pi_{3} \oplus \pi_{6} \oplus \pi_{4} \oplus 0, \\
& \text { dimensions } n(2 n-1) \times n(2 n-1) \\
& =\frac{1}{3} n(n+1)(2 n+1)(2 n-3)+\frac{1}{2} n(n+1)(2 n-1)(2 n-3) \\
& +(n+1)(2 n-1)+\frac{1}{6} n(n-1)(2 n-1)(2 n-3)+n(2 n-1)+1 . \\
& \\
E_{6}: & \pi_{2} \otimes \pi_{2}=2 \pi_{2} \oplus \pi_{6} \oplus\left(\pi_{1}+\pi_{3}\right) \oplus \pi_{2} \oplus 0, \\
& \text { dimensions } 78 \times 78=2430+2925+650+78+1 . \\
E_{7}: & \pi_{1} \otimes \pi_{1}=2 \pi_{1} \oplus \pi_{5} \oplus \pi_{4} \oplus \pi_{1} \oplus 0, \\
& \text { dimensions } 133 \times 133=7371+8645+1539+133+1 . \\
E_{8}: & \pi_{1} \otimes \pi_{1}=2 \pi_{1} \oplus \pi_{3} \oplus \pi_{2} \oplus \pi_{1} \oplus 0, \\
& \text { dimensions } 248 \times 248=27000+30380+3875+248+1 . \\
F_{4}: & \pi_{2} \otimes \pi_{2}=2 \pi_{2} \oplus \pi_{4} \oplus 2 \pi_{1} \oplus \pi_{2} \oplus 0, \\
& \text { dimensions } 52 \times 52=1053+1274+324+52+1 . \\
G_{2}: & \pi_{2} \otimes \pi_{2}=2 \pi_{2} \oplus 3 \pi_{1} \oplus 2 \pi_{1} \oplus \pi_{2} \oplus 0, \\
& \text { dimensions } 14 \times 14=77+77+27+14+1 .
\end{array}
$$

Acknowledgements. We are thankful to L. Michel for the important information that $\Delta$ occurs in $\Delta \otimes \Delta$ twice for $\mathrm{SU}(n), n \geqq 3$, and once for all other simple Lie algebras; and to Professor V. Singh for the suggestion that one should look for some transformation which distinguishes the $f$ from other possible solutions of Eq. (5.9). V. Singh also independently verified the reduction of $\Delta \otimes \Delta$ for the algebras $A_{n}$, $B_{n}, C_{n}$, and $D_{n}$ using Young tableaux and branching rules. M. L. Metha is grateful to C. Itzykson for a detailed discussion of Eq. (5.9) and to the Tata Institute of Fundamental Research, Bombay for its warm hospitality where this work germinated. V. Gupta is grateful to C.E.N. de Saclay for its hospitality which enabled us to finish the work.

\section{References}

1. Taylor, J.C.:Ward identities and charge renormalization of the Yang-Mills-field. Nucl. Phys. B33, $436(1971)$

Slavnov, A.: Ward identities in gauge theories. Theor. Math. Phys. 10, 99 (1972)

2. Dhar, A., Gupta, V.: In preparation (1982)

3. These are the symmetric and asymmetric momentum subtraction schemes discussed In: Dhar, A., Gupta, V.: Pramana 17, 469 (1981)

4. See for example, Hamermesh, M.: Group theory. Reading, MA: Addison-Wesley 1962, Sect. 5.6

5. Cartan, E.: Thesis (1894), reprinted in Oeuvres Complètes. Paris: Gauthier-Villars 1962 Part 1, Vol. 1;

Weyl, H.: Math. Z. 23, 271 (1925); 24, 328 (1926)

6. Mehta, M.L.: Classification of irreducible unitary representations of compact simple Lie groups. I. J. Math. Phys. 7, 1824 (1966)

Mehta, M.L., Srivastava, P.K.: Classification of irreducible unitary representations of compact simple Lie groups. II. J. Math. Phys. 7, 1833 (1966)

7. See for example, Macfarlane, A.J., Sudbery, A., Weisz, P.H.: On Gell-Mann's $\lambda$-matrices, $d$ - and $f$-tensors, octets, and parametrizations of SU(3). Commun. Math. Phys. 11, 77 (1968), for further useful relations involving $f$ and $d$

Communicated by H. Araki

Received September 20, 1982 